\title{
Black hole thermodynamics in Sharma-Mittal generalized entropy formalism
}

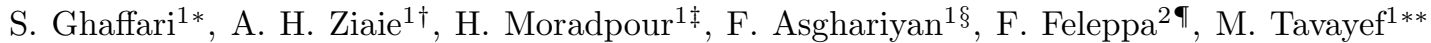 \\ ${ }^{1}$ Research Institute for Astronomy and Astrophysics of Maragha (RIAAM), P. O. Box 55134-441, Maragha, Iran \\ 2 Department of Physics, University of Trieste, via Valerio 2, 34127 Trieste, Italy
}

\begin{abstract}
Using the Sharma-Mittal entropy, we study some properties of the Schwarzschild and Schwarzschild-de Sitter black holes. The results are compared with those obtained by attributing the Bekenstein entropy bound to the mentioned black holes. Our main results show that while the Schwarzschild black hole is always stable in the micro-canonical ensemble, it can be stable in the canonical ensemble if its mass is bigger than the mass of the coldest Schwarzschild black hole. A semi-classical analysis has also been used to find an approximate relation between the entropy free parameters. Throughout the paper, we use units $c=G=\hbar=k_{B}=1$, where $k_{B}$ denotes the Boltzmann constant.
\end{abstract}

\section{INTRODUCTION}

The Jacobson's pioneering work [1] reveals the deep connections between gravity, spacetime and thermodynamics. It says that the system information (or equally the system entropy), combined with the thermodynamic laws, can address the gravitational field equations. The Bekenstein entropy bound is one of its argument pillars which also plays a crucial role in investigating the black hole thermodynamics [1 3]. This entropy is non-extensive, a property expected by considering the long-range nature of the gravity [4-11]. Indeed, the non-extensivity is also brought up in studying the thermodynamic properties of systems whenever the effects of the size of reservoir is considered [12, 13].

The above argument motivates physicists to study the cosmological and gravitational phenomena in the generalized statistical formalisms, such as non-extensive Rényi and Tsallis entropies, in which the ordinary probability distribution is replaced with a power-law distribution leading to generalized entropies [14 38]. It has been indicated that using the power-law distributions of probability is compatible with experimentally observed power-law tailed particle spectra [39-42]. Recently, using the Rényi entropy, as a single parameter generalized entropy [6, 9], the thermodynamic properties of some black holes has been studied [33 37]. It has been shown that the Schwarzschild black holes based on the Rényi formalism, in contrast to Boltzmann-Gibbs statistical mechanics, can be in stable equilibrium in the canonical ensemble [36, 37]. In spite of the successes of the non-extensive statistical mechanics, the non-extensive Tsallis entropy can not satisfy the zeroth law of thermodynamics without neglecting energy corrections [43].

On the other hand, its generalization, called the Sharma-Mittal entropy (SM) 9], which is a combination of the Rényi and Tsallis entropies, leads to interesting results in the cosmological setup. Such a kind of generalization helps us to describe the current accelerated universe by using the vacuum energy in a suitable manner [23 27]. Although, non-extensive entropies have been used to investigate the thermodynamic properties of black holes, but none of them consider the Sharma-Mittal entropy for it. All of these motivate us to study the thermodynamic properties of black holes, as strongly coupled gravitational systems, by employing the SM entropy written as $[9,23]$

$$
S_{S M}=\frac{1}{R}\left(\left(1+\delta S_{T}\right)^{\frac{R}{\delta}}-1\right) .
$$

Here, $S_{T}=\frac{A}{4}$, where $A=4 \pi r^{2}$ and $r$ denotes the horizon radius, is the Tsallis entropy [1, 23]. Moreover, $R$ and $\delta$ are free unknown parameters to be determined by fitting the results with observations [9, 23]. One can see that for $R \rightarrow 0$ and $R \rightarrow \delta$, the Rényi and Tsallis entropies are recovered, respectively.

It is worth mentioning that, there are two approaches to demonstrate the thermodynamic properties of black holes in the non-extensive statistical mechanics. In the first approach, similar to the standard picture of black hole thermodynamics, the temperature of a black hole is considered as the Hawking

\footnotetext{
* sh.ghaffari@riaam.ac.ir

$\dagger$ ah.ziaie@riaam.ac.ir

$\ddagger$ hn.moradpour@gmail.com

$\S$ fereshteh.asghariyan@yahoo.com

I feleppa.fabiano@gmail.com

** matin.tavayef@gmail.com
} 
temperature and so the energy of the system should be generalized. In another approach, the standard energy of the system is assumed and a corresponding generalized temperature associated with the generalized entropy can be obtained. In the present work, we use the second approach and find the generalized temperature associated with the Sharma-Mittal entropy to describe the thermodynamic properties of the Schwarzschild black hole.

In this paper, we are mainly interested to study some thermodynamic properties of the Schwarzschild (Sch) black holes meeting the SM entropy bound instead of the Bekenstein entropy bound. After addressing some general remarks of the SM entropy of black holes in the next section, we study the temperature and decay time of the Sch and Schwarzschild-de Sitter (SdS) black holes in the third section. A semi-classical approach is used, in the forth section, to find an approximate relation between $\delta$ and $R$ parameters of Sch black holes. Employing the Poincaré turning point method [2, 3, 36, 44], the stability of Sch black holes is also studied in both the micro-canonical and canonical ensembles in Sec. (V) where the heat capacity has also been investigated. The last section is also devoted to a summary. Throughout the paper, we also compare our results with those obtained by using the Bekenstein entropy.

\section{SM ENTROPY FOR BLACK HOLE HORIZON}

Consider a spherically symmetric static metric

$$
d s^{2}=f(\tilde{r}) d t^{2}-\frac{d \tilde{r}^{2}}{f(\tilde{r})}-\tilde{r}^{2} d \Omega^{2}
$$

where $t$ and $\tilde{r}$ are the time and radius coordinates, respectively, and $d \Omega^{2}=d \theta^{2}+\sin ^{2} \theta d \phi^{2}$ is the standard line element on a unit two-sphere. The Unruh temperature corresponding to the above metric horizon with radius $r$ obtained by solving $f(r)=0$ equation, is given as [45]

$$
T=\frac{1}{4 \pi} f^{\prime}(r)
$$

For a black hole with mass $M$ (the mass confined by radius $r$ ), one can use the Clausius formula to reach the entropy content $(S)$ of the black hole (with radius $r$ ) as [1]

$$
S=4 \pi \int_{0}^{M} \frac{d M}{f^{\prime}(r)}=4 \pi \int_{0}^{r} \int_{0}^{M} \delta(f(r, M)) d r d M,
$$

where we used the fact that $f^{\prime}(r)$ is a Jacobian for the Dirac-delta constraint [35] to write the last equality. In fact, the last equality addresses us a micro-canonical shell in a phase space whose variables are $r$ and $M(\equiv E)$ [35]. For the Bekenstein-Hawking entropy of the Sch black hole $\left(S_{B H}\right)$, where $f(r)=1-\frac{2 m}{r}$, we have

$$
S_{B H}=\pi r^{2}
$$

in which $r=2 M$ is the solution of $f(r)=0$. Therefore, simple calculations lead to 35$]$

$$
\begin{aligned}
& S_{B H}=4 \pi E^{2}, \quad \frac{1}{T_{B H}}=S_{B H}^{\prime}(E)=8 \pi E, \\
& C_{v}=-\frac{S_{B H}^{\prime}{ }^{2}(E)}{S_{B H}^{\prime \prime}(E)}=-8 \pi E^{2},
\end{aligned}
$$

where prime denotes derivative with respect to $E$. Moreover, $T_{B H}$ and $C_{v}$ are Hawking temperature and heat capacity of the black hole at constant volume, respectively [35].

\section{TEMPERATURE AND DECAY TIME FOR SCH AND SDS BLACK HOLES}

For the Sch black hole, for which $f(r)=1-2 M / r=0$, one can use Eqs. (6) along with Sharma-Mittal entropy (1) to obtain 


$$
\begin{aligned}
& S_{S M}(E)=\frac{1}{R}\left(\left(1+4 \delta \pi E^{2}\right)^{\frac{R}{\delta}}-1\right), \\
& \frac{1}{T_{S M}}=8 \pi E\left(1+4 \delta \pi E^{2}\right)^{\frac{R}{\delta}-1}
\end{aligned}
$$

and

$$
C_{v}=-\frac{8 \pi E^{2}\left(1+4 \delta \pi E^{2}\right)^{\frac{R}{\delta}}}{1+8 \pi R E^{2}-4 \delta \pi E^{2}}
$$

Now, using Eq. (7) one gets

$$
T_{S M}(r)=\frac{1}{4 \pi r}\left(1+\delta \pi r^{2}\right)^{1-\frac{R}{\delta}}
$$

as the Hawking temperature. In the limiting case $R=\delta$, the standard Hawking temperature is recovered. This limiting case also corresponds to Bekenstein-Hawking entropy which plays the role of the Tsallis entropy [19-21, 23, 31, 35-37, 43]. This result implies that the observed deviation from the Hawking temperature for the Schwarzschild black hole can be attributed to (or caused by) the non-extensive Sharma-Mittal entropy. For a SdS black hole, where $f(r)=1-2 M / r+\lambda r^{2} / 2$, in which $\lambda$ denotes the cosmological constant, the $f(r)=0$ equation yields

$$
M(r)=\frac{r}{2}\left(1+\lambda \frac{r^{2}}{2}\right),
$$

for the mass content of the horizon with radius $r$. Using the $E \equiv M$ definition together with Eqs. (8) and (9), the Hawking temperature is evaluated as

$$
T(r)=\frac{\partial M}{\partial S}=\frac{M^{\prime}(r)}{S^{\prime}(r)}=\frac{1+\frac{3 \lambda}{2} r^{2}}{4 \pi r\left(1+\delta \pi r^{2}\right)^{\frac{R}{\delta}-1}},
$$

where prime denotes derivative with respect to radius. In the limit of $R=\delta$, the standard temperature of Bekenstein-Hawking entropy for the SdS black hole [35] is regained. In Fig. 1] the temperature of Sch and SdS black holes have been plotted by using the above relations. The results of using the Bekenstein entropy have also been plotted for a better comparison.

Considering the Hawking radiation of a black hole as a black body radiation and bearing the Stefan-Boltzmann law in mind, one has

$$
\dot{M} \equiv \frac{d M}{d t}=A \sigma T^{4},
$$

where $\sigma$ is the Stefan-Boltzman constant, for the mass loss rate of a Schwarzschild black hole. It finally leads to

$$
\dot{M}=M^{\prime}(r) \dot{r}=-4 \pi r^{2} \sigma T^{4}(r),
$$

combined with Eq. (8) to obtain the decay time of the Sch black hole meeting the Sharma-Mittal entropy

$$
t_{s}=\frac{(4 \pi)^{3}}{\sigma} \int_{0}^{r} r^{2}\left(1+\delta \pi r^{2}\right)^{\frac{4 R}{\delta}-4} d r
$$

Hypergeometric functions are the solutions of the above integral. For the SdS black holes, calculations lead to

$$
d t_{s}=\frac{(4 \pi)^{3} r^{2}\left(1+\delta \pi r^{2}\right)^{\frac{4 R}{\delta}-4}}{\sigma\left(1+\frac{3}{2} \lambda r^{2}\right)^{3}} d r
$$

for the time decay, which yields again hypergeometric functions. Fig. 2 includes the $t_{s}(r)$ of the Sch and SdS black holes for both the Bekenstein and Sharma-Mital entropies. 


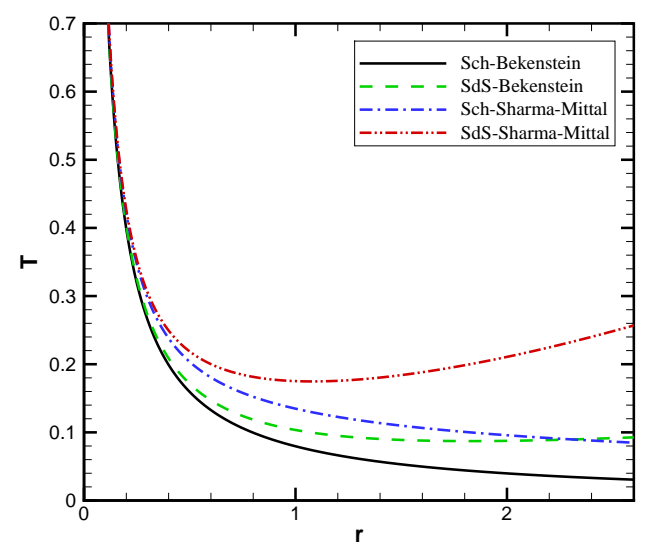

FIG. 1: Temperature $T$ versus the black hole radius $r$ for both the Bekenstein and Sharma-Mittal entropies. The values of $\lambda=-0.2, R=1.2$ and $\delta=1.8$ are adopted.

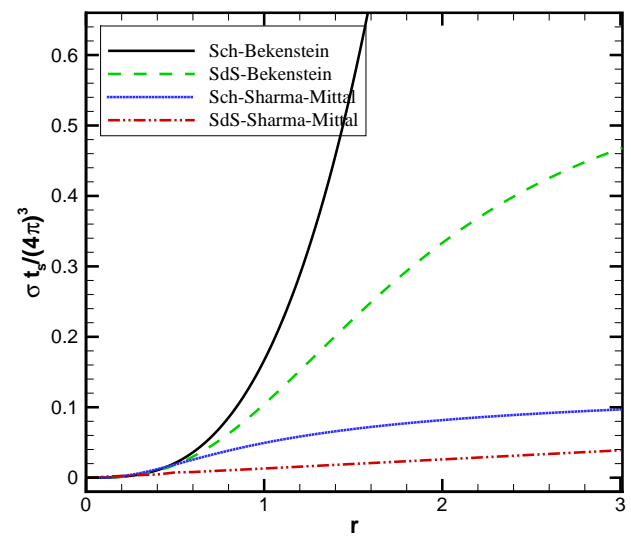

FIG. 2: $t_{s}(r)$ for both the Bekenstein and Sharma-Mittal entropies. We have set $\lambda=0.2, R=0.7$ and $\delta=1.5$.

\section{A SEMI-CLASSICAL INVESTIGATION FOR ENTROPY PARAMETERS OF SCH BLACK HOLES}

Consider a Sch black hole whose temperature is the minimum value of Eq. (8) leading to $r_{0}^{2}=\frac{1}{\pi(\delta-2 R)}$ for its radius. From classical point of view, its energy is $E_{0}=M\left(r_{0}\right)=\frac{r_{0}}{2}$. On the other hand, since this state has the minimum temperature, one may also approximate it with quantum mechanical considerations [35]. As a toy model, we look at this state as the ground state of a harmonic oscillator with energy $E=\frac{\omega}{2}=\frac{\pi}{\Lambda}$ (in the unit of $\hbar=c=1$ ). Here, $\Lambda$ denotes the wavelength of the system, and $2 r_{0}$ (the black hole diameter) is a suitable approach for $\Lambda$ [35] yielding $E \approx \frac{\pi}{2 r_{0}}$. Employing $E_{0} \approx E$ assumption, and bearing the $r_{0}^{2}=\frac{1}{\pi(\delta-2 R)}$ relation in mind, we reach

$$
\delta \approx \frac{1}{2 \pi^{2}}+2 R
$$

as an approximate relation between $\delta$ and $R$ in the mentioned situation. 


\section{STABILITY OF SCH BLACK HOLE}

Here, we are going to study the stability of Sch black holes, meeting the Sharma-Mittal entropy bound, in both the micro-canonical [2, 3, 36, 44] and canonical [12, 13, 36] approaches. It has been shown that in the BoltzmannGibbs picture the Sch black holes cannot be in stable equilibrium which implies that the canonical ensemble can not be used when the gravitational interaction is included in the standard statistical mechanics [46, 47]. In the micro-canonical framework, it has been assumed that the Sch black hole is isolated, and thus, $\beta$ and $M$ are the corresponding conjugate thermodynamic variables which have the mutual relation $[2,3,3,36,44]$

$$
\beta=\frac{\partial S}{\partial E},
$$

where $S$ denotes the system entropy. Hence, $\beta=\frac{1}{T}$ and

$$
\beta=8 \pi M\left(1+4 \delta \pi M^{2}\right)^{\frac{R}{\delta}-1},
$$

which recovers the result of employing the Bekenstein entropy $(\beta=8 \pi M)$ at the appropriate limit $R=\delta$ [36]. As long as the slope of the $\beta$ curve (versus $M$ ) is not vertical, the configuration under study does not admit any turning point meaning that the system remains stable [2, 3, 36, 44]. In Fig. 3, $\beta$ versus $M$ has been plotted for both the Bekenstein and Sharma-Mittal entropies indicating that the isolated Sch black hole, similarly to the standard Boltzmann-Gibbs picture, is always stable against spherically symmetric perturbations in both cases (no turning point is observed).

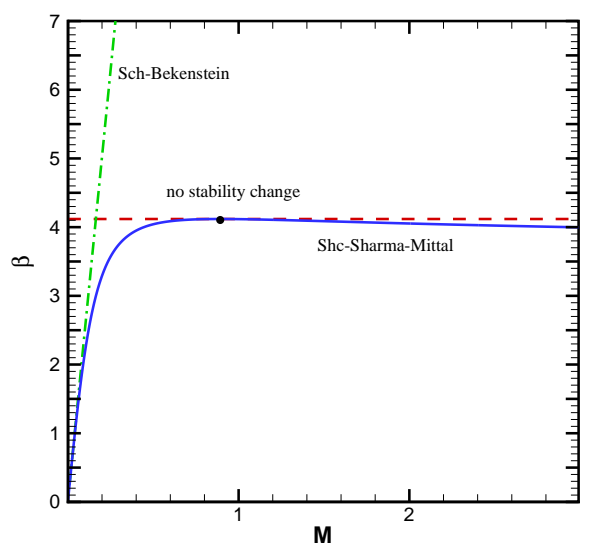

FIG. 3: The $\beta(M)$ curves of a Sch black hole within the frameworks of Bekenstein and Sharma-Mittal entropies in the microcanonical regime when $R=0.7$ and $\delta=1.5$. No vertical tangent occurs in either case.

The Helmholtz free energy formalism $(F)$ is the backbone of the canonical survey of a black hole in contact with a thermal bath, which is defined as $[12,13,36]$

$$
F=\frac{S}{\beta}-M
$$

where $\beta$ and $-M\left(\equiv \frac{d(\beta F)}{d \beta}\right)$ are the conjugate thermodynamic variables [12, 13, 36]. As it is apparent from Fig. 4, the tangent of the $-M(\beta)$ curve is vertical when $M=M\left(r_{0}\right) \equiv M_{0}$, or equally, the temperature of the Sch black hole is the minimum value of Eq. (8) $\left(T_{0}=1 / \sqrt{\pi(\delta-2 R)}\right)$. This means that, Schwarzschild black hole in the Sharma-Mittal formalism, in contrast to the Bekenstein-Hawking entropy, can be stable for $M>M_{0}$. We therefore conclude that black holes with $M>M_{0}$ have positive specific heat capacity and are stable while those with $M<M_{0}$ are unstable. These results are comparable with those obtained 


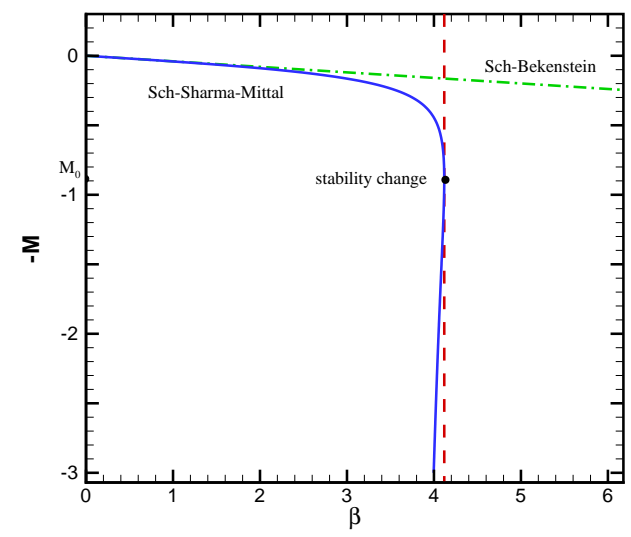

FIG. 4: The $-M(\beta)$ curves of a Sch black hole within the Boltzmann and Sharma-Mittal frameworks in the canonical ensemble when $R=0.7$ and $\delta=1.5$.

\section{in [36] for the Schwarzschild black holes described by the Rényi formalism.}

The sign of the heat capacity $C\left(\equiv \frac{d M}{d T}\right)$ can also help us in determining the stability of the black holes (the Hessian analysis) [36]. From Fig. 5, one can see that, unlike the Bekenstein formalism, the sign of the heat capacity is changed at $M_{0}$ point whenever the Sharma-Mittal entropy is attributed to the Sch black hole. Indeed, for $M>M_{0}$, the heat capacity is positive and meaningful, while for $M<M_{0}$, the heat capacity is negative meaning that such Sch black holes violate the laws of thermodynamics, a result obtainable in the Bekenstein formalism for which the heat capacity of Sch black hole is always negative.

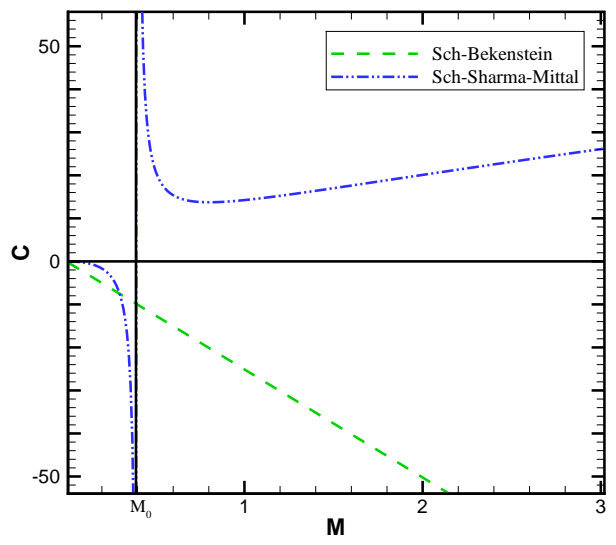

FIG. 5: Heat capacity of Sch black hole for both the Bekenstein and Sharma-Mittal entropies. Here, $R=0.6$ and $\delta=1.8$.

\section{CONCLUSION}

Our main focus, in this work, was to study some properties of a Sch black hole meeting the Sharma-Mittal entropy and compare them with those obtained by employing the Bekenstein entropy. Firstly, we addressed a time that the Sch and SdS black holes need to be completely evaporated through the Hawking radiation mechanism. Considering 
a Sch black hole with minimum temperature $\left(T_{0}\right)$ together with using a semi-classical analysis, we could obtain a bound on the entropy parameters $\delta$ and $R$. It has also been found out that, in the Sharma-Mittal formalism, a Sch black hole has positive heat capacity and is stable in the canonical ensemble framework, if its mass is bigger than $M_{0}$ which is the mass of a Sch black hole with temperature $T_{0}$. This result is contrary to the standard Boltzmann-Gibbs statistical mechanics. The stability analysis also shows that similar to the Bekenstein case, a Sch black hole meeting the Sharma-Mittal entropy bound is always stable in the framework of micro-canonical ensemble.

\section{Acknowledgments}

The work of S. Ghaffari has been supported financially by Research Institute for Astronomy and Astrophysics of Maragha (RIAAM).

[1] T. Jacobson, Phys. Rev. Lett. 75, 1260 (1995).

[2] O. Kaburaki, I. Okamoto, J. Katz, Phys. Rev. D 47, 2234 (1993);

J. Katz, I. Okamoto, O. Kaburaki, Class. Quant. Gravit. 10, 1323 (1993);

O. Kaburaki, Gen. Relativ. Gravit. 28, 843 (1996).

[3] G. Arcioni, E. Lozano-Tellechea, Phys. Rev. D 72, 104021 (2005).

[4] M. Gell-Mann, C. Tsallis, Nonextensive Entropy-Interdisciplinary Applications (Oxford University Press, New York, 2004).

[5] S. Abe, Foundations of Nonextensive Statistical Mechanics. In: Sengupta A. (eds) Chaos, Nonlinearity, Complexity. Studies in Fuzziness and Soft Computing 206. (Springer, Berlin, Heidelberg 2006).

[6] A. Rényi, Probability Theory (North-Holland, Amsterdam, 1970).

[7] S. Abe, Phys. Rev. E 63, 061105 (2001).

[8] H. Touchette, Physica A 305, 84 (2002).

[9] M. Masi, Phys. Lett. A 338, 217 (2005) .

[10] T. S. Biró, P. Ván, Phys. Rev. E 83, 061147 (2011).

[11] A. Majhi, Phys. Lett. B 775, 32 (2017).

[12] T. S. Biró, Physica A 392, 3132 (2013).

[13] T. S. Biró, et al., Eur. Phys. J. A 49, 110 (2013).

[14] E. M. C. Abreu, J. Ananias Neto, A. C. R. Mendes, W. Oliveira, Physica. A 392, 5154 (2013).

[15] E. M. C. Abreu, J. Ananias Neto. Phys. Lett. B 727, 524 (2013).

[16] E. M. Barboza Jr., R. C. Nunes, E. M. C. Abreu, J. A. Neto, Physica A: Statistical Mechanics and its Applications, 436, $301(2015)$.

[17] H. Moradpour, Int. Jour. Theor. Phys. 55, 4176 (2016).

[18] R. C. Nunes, et al. JCAP, 08, 051 (2016).

[19] N. Komatsu, Eur. Phys. J. C 77, 229 (2017).

[20] H. Moradpour, A. Bonilla, E. M. C. Abreu, J. A. Neto, Phys. Rev. D 96, 123504 (2017).

[21] V. G. Czinner and H. Iguchi, Phys. Lett. B 752, 306 (2016).

[22] V. G. Czinner and F. C. Mena, Phys. Lett. B 758, 9 (2016).

[23] A. Sayahian Jahromi, et al., Phys. Lett. B 780, 21 (2018).

[24] E.C. G. Demirel, Can. J. Phys. DOI:10.1139/cjp-2018-0784

[25] A. Jawad, et al., Symmetry, 10, 635 (2018).

[26] E. Sadri, M. Khurshudyan, arXiv:1809.07595 2.

[27] M. Younas, et al., AHEP, 2019, 1287932 (2019).

[28] M. Tavayef, A. Sheykhi, K. Bamba, H. Moradpour, Phys. Lett. B 781, 195 (2018).

[29] S. Ghaffari et al., Phys. Dark. Univ. 23, 100246 (2019).

[30] H. Moradpour, A. Sheykhi, C. Corda, I. G. Salako, Phys. Lett. B 783, 82 (2018).

[31] H. Moradpour, et al. Eur. Phys. J. C 78, 829 (2018).

[32] E. M. C. Abreu et al., Eur. Phys. Lett. 124, 30003 (2018).

[33] A. Bialas, W. Czyz, Euro. Phys. Lett. 83, 60009 (2018).

[34] A. Belin, A. Maloney, S. Matsuura, arXiv:1306.2640.

[35] T. S. Biró, V. G. Czinner, Phys. Lett. B 726, 861 (2013).

[36] V. G. Czinnera, H. Iguchia, Phys. Lett. B 752, 306 (2016).

[37] V. G. Czinnera, H. Iguchia, Eur. Phys. J. C 77, 892 (2017).

[38] Tsallis C., Introduction to Non-Extensive Statistical Mechanics: Approaching a Complex World, Springer, (2009).

[39] Tsallis C., J. Stat. Phys. 52 (1988) 50.

[40] Tsallis C., Physica A 221(1995) 277. 
[41] Tsallis C., Braz. J. Phys. 29 (1999) 1.

[42] Tsallis C., Eur. Phys. J. A 40 (2009) 257.

[43] Q.A. Wang, Euro. Phys. J. B 26, 357 (2002);

Q.A. Wang and A. Le Méhauté, J. Math. Phys. 43, 5079 (2002).

[44] H. Poincara, Acta. Math. 7, 259 (1885).

[45] W. G. Unruh, Phys. Rev. D 14, 870 (1976).

[46] S.W. Hawking, Black hole explosions. Nature, 248 30, (1974).

[47] S.W. Hawking, Don N. Page, Thermodynamics of Black Holes in Anti-de Sitter Space, Commun. Math. Phys. 87, 577 (1983). 